\title{
Efeitos do Uso de Crotalaria juncea e Carbofuran em Fitonematóides Ectoparasitos de Cana-de-Açúcar
}

\author{
Regina Ceres T. da Rosa*, Romero M. de Moura* \& Elvira Maria R. Pedrosa* \\ Departamento de Agronomia, Universidade Federal Rural de Pernambuco, Dois Irmãos, CEP 52171-900, Recife, PE, \\ e-mails: reginactrosa@ubbi.com.br, romeromoura@yahoo.com.br
}

(Aceito para publicação em 02/02/2004)

Autor para correspondência: Romero Marinho de Moura

ROSA, R.C.T., MOURA, R.M. \& PEDROSA, E.M.R. Efeitos do uso de Crotalaria juncea e carbofuran em fitonematóides ectoparasitos de cana-de-açúcar. Fitopatologia Brasileira 29:447-449. 2004.

\section{RESUMO}

Foi estudado comparativamente o efeito do cultivo de Crotalaria juncea, por um ano, com incorporação, a uma aplicação do nematicida sistêmico carbofuran, no momento do plantio, em cana-de-açúcar (Saccharum sp.), var. SP79-1011, em solos naturalmente infestados por Helicotylenchus dihystera, Criconemella ornata, Paratrichodorus minor e Trichodorus sp. Foram determinados os níveis populacionais dos nematóides, acompanhados ao longo do experimento, e o comportamento de cada um no solo. As populações dos fitonematóides ectoparasitos estudados apresentaram número reduzido de espécimes, sendo pouco influenciadas pela incorporação da crotalária ou da aplicação do nematicida. Todas as populações permaneceram baixas, ao longo do desenvolvimento da planta, observando-se pequeno incremento populacional a partir dos 90 dias. O comportamento da população de $C$. ornata em função do tempo nas parcelas tratadas com crotalária ou carbofuran foi expresso pelas equações $\mathrm{Y}=100,56524+$ $0,01504 \mathrm{X}-0,00003032 \mathrm{X}^{2}\left(\mathrm{R}^{2}=0,5605^{* *}\right)$ ou $\mathrm{Y}=10^{0,62059+0,01394 \mathrm{X}-0,00002786 \mathrm{X}^{2}}$ $\left(\mathrm{R}^{2}=0,6416^{* *}\right)$, respectivamente. Ainda, em cana tratada com carbofuran, o aumento populacional de Trichodorus sp., no decorrer do experimento, obecedeu ao modelo quadrático $\mathrm{Y}=13,16667$ $0,10904 \mathrm{X}+0,00065711 \mathrm{X} 2\left(\mathrm{R}^{2}=0,6916^{* *}\right)$.

Palavras-chave adicionais: Saccharum sp., Helicotylenchus dihystera, controle, Criconemella ornata, Paratrichodorus minor Trichodorus sp., dinâmica de população.

\section{ABSTRACT}

Effects of Crotalaria juncea and carbofuran on ectoparasite nematodes of sugarcane

A study was done on the effects of Crotalaria juncea and carbofuran on ectoparasite nematodes of sugarcane (Saccharum sp.) planted in soil naturally infested by Helicotylenchus dihystera, Criconemella ornata, Paratrichodorus minor, and Trichodorus sp. The stydy compared the use of $C$. juncea for one year, with incorporation, with one application of the systemic nematicide carbofuran at time of planting sugarcane var. SP79-1011. Evaluations were based upon soil nematode population densities throughout the first year of cropping. The nematode populations

Em canaviais do mundo inteiro, os nematóides endoparasitos sedentários, com freqüência, são reconhecidos como indutores da maior parte das perdas de produção agrícola causadas por fitopatógenos. Contudo, vários ectoparasitos patogênicos a muitas culturas, das mais variadas famílias botânicas, em regiões especificas, também têm sido encontrados associados à cana-de-açúcar (Saccharum sp.) (Luc et al., 1990; Starr \& Bendezu, 2002). Em híbridos de cana-deaçúcar os nematóides têm sido assinalados em raízes e solo de rizosfera e mais de 275 espécies de 48 gêneros já foram registrados, sendo os ectoparasitos os mais freqüentes e abundantes (Novaretti et al., 1974; Cadet \& Spaull, 1985; Moura, 1991;1995). No Brasil, a patogenicidade dos ectopara-

\section{*Bolsista do CNPq}

showed low number of specimens, being slighly influenced by the crotalaria incorporation and carbofuran application. Nematode population level remained low, during plant development, with low increment, in general, after 90 days of planting. The behavior of the C. ornata population in function of the time in plots treated with crotalaria or carbofuran was described by the equations $\mathrm{Y}=$ $100,56524+0,01504 \mathrm{X}-0,00003032 \mathrm{X}^{2}\left(\mathrm{R}^{2}=0,5605^{* *}\right)$ or $\mathrm{Y}=10^{0,62059+}$ $0,01394 \mathrm{X}-0,00002786 \mathrm{X}^{2}\left(\mathrm{R}^{2}=0,6416^{* *}\right)$, respectively. On sugarcane treated with carbofuran the increase of Trichodorus sp. population followed the quadractic model $\mathrm{Y}=13,16667-0,10904 \mathrm{X}+$ $0,00065711 \mathrm{X}^{2}\left(\mathrm{R}^{2}=0,6916^{* *}\right)$. sitos ainda não foi pesquisada, muito embora existam estudos mostrando associações quase permanentes entre cana-deaçúcar e os gêneros Helicotylenchus Steiner, Paratrichodorus Siddiqi, Trichodorus Cobb, Tylenchorhynchus Cobb, Hemicycliophora De Man, Xiphinema Cobb e Criconemella De Grisse \& Loof (Cruz et al., 1986; Moura et al., 1999; Moura, 2000 e Moura et al., 2000). O objetivo do presente trabalho foi avaliar, comparativamente, em cana-planta, o efeito de Crotalaria juncea L. e do nematicida sistêmico carbofuran, sobre as populações dos ectoparasitos Helicotylenchus dihystera (Cobb) Sher, Criconemella ornata De Grisse \& Loof, Paratrichodorus minor (Colbran) Siddiqi e Trichodorus sp.

O experimento foi desenvolvido em canaviais da destilaria Miriri, no Estado da Paraíba, num período de dois 
anos (maio/2000 a junho/2002), em solos arenosos, cultivados há muitos anos com cana-de-açúcar. Inicialmente, nas Fazendas Santa Terezinha I e Santa Emília I, uma grande área foi preparada para o plantio com cana-de-açúcar variedade SP79-1011. Áreas adjacentes, do mesmo campo, foram cultivadas com C. juncea, por 12 meses (maio/2000 a maio/2001), usando-se incorporação, a cada ciclo, ou seja, cada três meses. Após o último ciclo da crotalária, todas as áreas foram colhidas e preparadas, de modo convencional, para cultivo com canade-açúcar SP79-1011 (junho/2001 a junho/2002). Antes, porém, para execução da pesquisa, foram estabelecidos em cada fazenda três tratamentos experimentais, cada um com três repetições. Cada repetição era constituída por três linhas de $8,3 \mathrm{~m}$ de comprimento, locais onde foram coletados aleatoriamente todos os dados da pesquisa. Os tratamentos foram caracterizados pelo plantio da cana-de-açúcar SP791011, em: 1) solo previamente cultivado com cana-de-açúcar; 2) solo previamente cultivado com $C$. juncea; 3) solo previamente cultivado com cana-de-açúcar SP79-1011, seguindo-se aplicação do nematicida sistêmico carbofuran, no fundo do sulco, na dose de $60 \mathrm{~kg}$ i.a./ha no momento do plantio. $\mathrm{Na}$ elaboração do desenho experimental, formaram-se dois blocos, sendo a Fazenda Santa Terezinha I considerada o Bloco 1 e Santa Emília I Bloco 2, com três repetições dentro dos blocos. Durante o experimento, foram feitas determinações dos índices populacionais dos ectoparasitos aos 45, 90, $180 \mathrm{e}$ 360 dias após o plantio da cana-de-açúcar. Para tal fim, foram coletadas, nas repetições, cinco subamostras por linha, em ziguezague, totalizando nove amostras compostas por bloco. Os níveis foram determinados em alíquotas de $300 \mathrm{cc}$ de solo, processadas pelo método de Jenkins (1964). Os dados relativos foram transformados em $\log _{10}(x+1)$, para análise de variância, empregando-se o teste de Tukey a $5 \%$ de probabilidade para comparação das médias dos tratamentos. Com os dados obtidos ao longo do período experimental foram testados modelos lineares, quadráticos e logarítmicos, objetivando verificar qual descreveria melhor o comportamento das populações no solo, durante o período de cultivo da cana-de-açúcar, nas condições utilizadas.

Ao longo do experimento, registrou-se índice pluviométrico médio da ordem de 179,2 $\mathrm{mm}$, com amplitude de 1-584 mm (Figura 1), e as populações dos fitonematóides apresentaram números reduzidos de espécimes (Tabela 1). É possível que esses baixos índices populacionais estejam relacionados com as baixas médias pluviométricas da região, nos meses correspondentes às coletas dos dados experimentais efetuadas nos períodos de 90 e 180 dias (Figura 1). Conforme conhecido, os ectoparasitos completam integralmente o ciclo de vida na rizosfera, sofrendo influência direta da planta hospedeira e, principalmente, das condições ambientais,

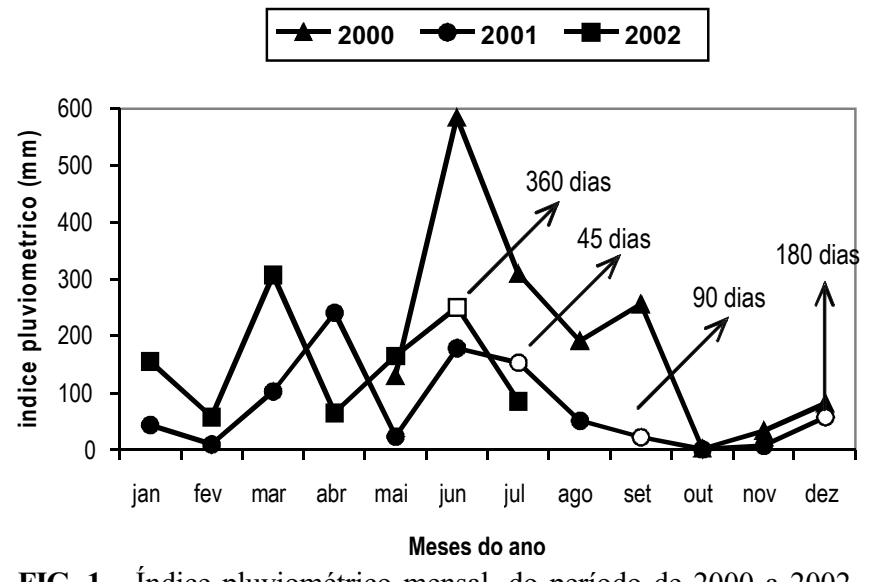

FIG. 1 - Índice pluviométrico mensal, do período de 2000 a 2002, observado na Destilaria Miriri, Mamanguape, PB. As marcas em branco indicam os momentos das coletas das amostras em número de dias após o plantio.

TABELA 1 - Índices populacionais de nematóides ectoparasitos em solo cultivado com cana-de-açúcar variedade SP79-1011, durante um período de 12 meses

\begin{tabular}{|c|c|c|c|c|c|}
\hline \multirow{3}{*}{ Dias } & \multirow{3}{*}{ Tratamento $^{1}$} & \multicolumn{4}{|c|}{ Nematóide } \\
\hline & & \multicolumn{4}{|c|}{ Solo $\left(100 \mathrm{~cm}^{3}\right)$} \\
\hline & & Helicotylenchus dihystera & Criconemella ornata & Trichodorus $s p$. & Paratrichodorus minor \\
\hline \multirow{3}{*}{45} & Crotalaria juncea & $14,5 \mathrm{a}$ & $12,7 \mathrm{a}$ & $0,50 \mathrm{~b}$ & $0,33 \mathrm{a}$ \\
\hline & Carbofuran & $4,00 \mathrm{a}$ & $19,3 \mathrm{a}$ & $0,83 \mathrm{~b}$ & $0,33 \mathrm{a}$ \\
\hline & Testemunha & $11,0 \mathrm{a}$ & $42,0 \mathrm{a}$ & $7,67 \mathrm{a}$ & $1,83 \mathrm{a}$ \\
\hline \multirow{3}{*}{90} & Crotalaria juncea & $32,8 \mathrm{a}$ & $85,5 \mathrm{a}$ & $31,2 \mathrm{a}$ & $15,7 \mathrm{a}$ \\
\hline & Carbofuran & $13,0 \mathrm{a}$ & $71,7 \mathrm{a}$ & $24,0 \mathrm{a}$ & $10,3 \mathrm{a}$ \\
\hline & Testemunha & $27,7 \mathrm{a}$ & $47,5 \mathrm{a}$ & $46,5 \mathrm{a}$ & $16,0 \mathrm{a}$ \\
\hline \multirow{3}{*}{180} & Crotalaria juncea & $46,8 \mathrm{a}$ & $177,3 \mathrm{a}$ & $6,17 \mathrm{a}$ & $1,33 \mathrm{~b}$ \\
\hline & Carbofuran & $32,3 \mathrm{ab}$ & $146,2 \mathrm{a}$ & $7,17 \mathrm{a}$ & $9,67 \mathrm{a}$ \\
\hline & Testemunha & $12,3 \mathrm{~b}$ & $68,50 \mathrm{~b}$ & $17,0 \mathrm{a}$ & $3,17 \mathrm{ab}$ \\
\hline \multirow{3}{*}{360} & Crotalaria juncea & $40,0 \mathrm{a}$ & $192,7 \mathrm{a}$ & $18,7 \mathrm{~b}$ & $12,2 \mathrm{a}$ \\
\hline & Carbofuran & $22,7 \mathrm{a}$ & $135,5 \mathrm{a}$ & $60,7 \mathrm{a}$ & $10,5 \mathrm{a}$ \\
\hline & Testemunha & $22,7 \mathrm{a}$ & $122,2 \mathrm{a}$ & $10,0 \mathrm{~b}$ & $5,00 \mathrm{a}$ \\
\hline
\end{tabular}

Para análise estatística os dados foram transformados para $\log _{10}(x+1)$, estando apresentadas médias, de três repetições para cada tratamento, dos dados originais.

${ }^{1}$ Em cada coluna, média seguida de letras distintas são diferentes entre si ao nível de 5\% de probabilidade pelo teste de Tukey. 
Efeitos do uso de Crotalaria juncea e carbofuran em fitonematóides...

especialmente precipitação, temperatura e propriedades físicoquímicas do solo (Novaretti \& Nelli, 1980; Carneiro et al., 1980; Kandji et al., 2001). Em estudos recentes, Barros et al. (2002) e Chaves et al. (2003) verificaram baixas densidades populacionais de ectoparasitos associados à cana-de-açúcar (planta e soca) nas condições do Nordeste brasileiro estando de acordo com os dados presentes. Os níveis dos quatro gêneros constatados no presente estudo não foram reduzidos pela incorporação da crotalária nem pela aplicação do carbofuran antes do plantio (Tabela 1). Também, foram pouco influenciados pela idade da planta, observando-se pequeno incremento populacional desses parasitos a partir dos 90 dias. Criconemella ornata apresentou as maiores densidades populacionais. O comportamento da população de $C$. ornata em função do tempo nas parcelas tratadas com crotalária ou carbofuran foi expresso pelas equações $\mathrm{Y}=100,56524+0,01504 \mathrm{X}$ $0,00003032 \mathrm{X}^{2}\left(\mathrm{R}^{2}=0,5605^{* *}\right)$ ou $\mathrm{Y}=10^{0,62059+0,01394 \mathrm{X}-0,00002786 \mathrm{X}^{2}}\left(\mathrm{R}^{2}\right.$ $\left.=0,6416^{* *}\right)$ respectivamente. Ainda, em cana tratada com carbofuran, o aumento populacional de Trichodorus sp., no decorrer do experimento, obecedeu ao modelo quadrático $\mathrm{Y}$ $=13,16667-0,10904 \mathrm{X}+0,00065711 \mathrm{X}^{2}\left(\mathrm{R}^{2}=0,6916^{* *}\right)$ (Figura 2). Para as demais combinações de tratamentos e nematóides não foram encontrados modelos lineares, quadráticos ou logarítmicos adequadamente ajustados que descrevessem a flutuação populacional dos parasitos nas condições estudadas.

\section{REFERENCIAS BIBLIOGRAFICAS}

BARROS, A.C.B., MOURA, R.M. \& PEDROSA, E.M.R. Efeito da aplicação de terbufos nas populações de três fitonematóides ectoparasitos em cana-de-açúcar. Fitopatologia Brasileira 27:309-311. 2002.

CADET, P. \& SPAULL, V.W. Studies on the relationship between nematodes and sugarcane in South and West África: plant cane. Revue de Nématologie 8:131-142. 1985.

CARNEIRO, R.M.D.G., LORDELLO, L.G.E., CARNEIRO, R.G. \& MONTEIRO, A.R. Flutuação populacional de quatro espécies de nematóides parasitos da cana-de-açúcar (Saccharum officinarum L.) em relação à pluviosidade e umidade do solo. Nematologia Brasileira 4:127-141. 1980 .
CHAVES, A., PEDROSA, E.M.R. \& MOURA, R.M. Efeito de terbufos em soqueira sobre fitonematóides ectoparasitos de cana-deaçúcar. Fitopatologia Brasileira 28:195-198. 2003.

CRUZ, M.M., SILVA, S.M.S. \& RIBEIRO, A.G. Levantamento populacional de nematóides em cana-de-açúcar em áreas de baixa produtividade nos Estados de Alagoas e Sergipe. Nematologia Brasileira 10:27-28. 1986.

JENKINS, W.R. A rapid centrifugal-flotation technique for separating nematodes from soil. Plant Disease Reporter 48:292. 1964.

LUC, M., SIKORA, R.A. \& BRIDGE, J. Plant Parasitic Nematodes in Subtropical and Tropical Agricuture. Wallingford. CABI Publishing. 2002.

MOURA, R.M. Dois anos de rotação de culturas em campos de canade-açúcar para controle de meloidoginose. 1. efeito dos tratamentos na população do nematóide. Nematologia Brasileira 15:1-7.1991.

MOURA, R.M. Dois anos de rotação de culturas em campos de canade-açúcar para controle de meloidoginose. 2. considerações sobre o método e reflexos na produtividade agroindustrial da cana-planta. Fitopatologia Brasileira 20:597-600. 1995.

MOURA, R.M. Controle integrado dos nematóides da cana-de-açúcar no Nordeste do Brasil. Anais, $22^{\circ}$ Congresso Brasileiro de Nematologia, Uberlândia, MG. 2000. pp. 88-94.

MOURA, R.M., PEDROSA, E.M.R., MARANHÃO, S.R.V.L., MOURA, A.M., MACEDO, M.E.A. \& SILVA, E.G. Nematóides associados à cana-de-açúcar no Estado de Pernambuco, Brasil. Nematologia Brasileira 23:92-99. 1999.

MOURA, R.M., PEDROSA, E.M.R., MARANHÃO, S.R.V.L., MACEDO, M.E.A., MOURA, A.M., SILVA, E.G. \& LIMA, R.F. Ocorrência dos nematóides Pratylenchus zeae e Meloidogyne spp. em cana-de-açúcar no Nordeste do Brasil. Fitopatologia Brasileira 25:101-103. 2000

NOVARETTI, W.R.T., ROCCIA, A.D., LORDELLO, L.G.E. \& MONTEIRO, A.R. Contribuição ao estudo dos nematóides que parasitam a cana-de-açúcar em São Paulo. Resumos, $1^{\text {a }}$ Reunião Brasileira de Nematologia, Piracicaba, SP. 1974. pp.21-32.

NOVARETTI, W.R.T. \& NELLI, E.J. Flutuações populacionais de nematóides na cultura de cana-de-açúcar, cana de ano e meio. Brasil Açucareiro 96:30-36. 1980.

STARR, J.L. \& BENZEDU, I.F. Ectoparasitos nematodes. In: Starr, J.L., Cook, R. \& Bridge, J. (Eds.) Plant Resistance to Parasitic Nematodes. Wallingford. CABI Publishing. 2002. pp.229-239. 